\title{
DEVELOPING A CONVECTIVE CODE WITH VORTICITY TRANSPORT IN UPWIND METHOD
}

\author{
Je -Ee Ho \\ Department of Mechanical Engineering, National Ilan University, Ilan, Taiwan, R.O.C., jeho@niu.edu.tw
}

Follow this and additional works at: https://jmstt.ntou.edu.tw/journal

Part of the Mechanical Engineering Commons

\section{Recommended Citation}

Ho, Je -Ee (2008) "DEVELOPING A CONVECTIVE CODE WITH VORTICITY TRANSPORT IN UPWIND METHOD," Journal of Marine Science and Technology. Vol. 16: Iss. 3, Article 4.

DOI: 10.51400/2709-6998.2004

Available at: https://jmstt.ntou.edu.tw/journal/vol16/iss3/4

This Research Article is brought to you for free and open access by Journal of Marine Science and Technology. It has been accepted for inclusion in Journal of Marine Science and Technology by an authorized editor of Journal of Marine Science and Technology. 


\title{
DEVELOPING A CONVECTIVE CODE WITH VORTICITY TRANSPORT IN UPWIND METHOD
}

\author{
$\mathrm{Je}-\mathrm{Ee} \mathrm{Ho}^{*}$
}

Key words: upwind scheme.

\begin{abstract}
The Navier-Strokes equations are usually used to solve the convective problems, however, to satisfy the conversation of mass, the uncertainty of pressure terms will easily trigger off the numerical divergence during the iterative process; which results in a calculating failure and makes the numerical solutions impossible. In this study, a vorticity-transport method that avoids the involvement of pressure terms by cross differentiate the governing equations and meets the flow conservation with stream function was proposed. With the aid of the upwind scheme and implicit method, an effective transformation from the partial differential equations into the vortex potential algebraic equations would be completed. From the numerical solution showed, not only agreed with the experiment result and the simulation of CFD package "NISTIR", but also the available scope of Reynold number and Pelect number could be extended up to 2500,25 which had approached to the turbulent transition.
\end{abstract}

\section{INTRODUCTION}

The computational code on fluid dynamics has been widely used as a popular simulation tools in convective research or engineering application. However, due to easy way and effective performance in running package, the development in numerical scheme becomes less attracted and gradually ignored today

Chorin [3] used vorticity concept to combine flow and temperature fields to form a system of simultaneous equations, which were solved with the discrete vortex method and yielded a low Reynold theoretical solution. Patanlcar and Spadling [6] developed a generalized formulation of auxiliary function for various schemes, such as hybrid scheme, exponential scheme and power scheme. With it, the degree of satisfactoriness of each function in simulating the flow and temperature had been further discussed by comparison with the exact solution. A variation of Mac Cormack method, upwind method, was first

Paper submitted 05/15/07; accepted 09/19/07. Author for correspondence: Je-EeHo (e-mail:jeho@niu.edu.tw).

*Department of Mechanical Engineering, National Ilan University, Ilan, Taiwan, R.O.C. introduced by Warming and Beam [8]. By using it, iterative procedure was divided into both predictor, corrector steps and the convergent solution with second-order truncation error would be achieved, Furthermore, a amplification factor with analysis in relative error to predict the stability condition was also successfully derived through the backward upwind scheme. Ames [1] applied Successive Over-Relaxation (SOR) technology to accelerate the iterative procedure, in which over-relaxation $(\alpha>1.0)$ was taken to be appropriate for Laplace's equation with Dirichlet boundary conditions while the under-relaxation was considered to the elliptic problem or nonlinear equations. To maintain diagonal dominance in the application of Thomas algorithm, a restricted $\omega$ variable, $\omega \leq 1+(\Delta x / \Delta y)^{2}$, was specified to ensure the convergent solution. Chilukuri and Pletcher [2] used the central-difference method to solve successfully the simultaneous equations. But the explicit way with the number of numerical solution solved one by one was applied in their calculating process, so the rate of convergence was low and the Reynold number had to be restricted to a low range.

In the present study, we transform the flow field equations into the vorticity - stream form and remain the temperature field unchanged. With the discretization by finite difference, the first order derivatives are represented by the upwind scheme and the central-difference is required in second order derivatives; so that the flow field can be converted to an elliptic problem and further discretized as algebra simultaneous equations. In this way, a vorticity - stream field becomes a simple boundary value problem that can be numerically solved more easily and used for a wider range of Reynold number.

\section{Dimensionless Governing Equation}

Mass conservation :

$$
\frac{\partial u}{\partial x}+\frac{\partial v}{\partial y}=0
$$

Moment equation:

$$
\begin{aligned}
& x: \quad u \frac{\partial u}{\partial x}+v \frac{\partial u}{\partial y}=-\frac{\partial p}{\partial x}+\frac{1}{\operatorname{Re}}\left(\frac{\partial^{2} u}{\partial x^{2}}+\frac{\partial^{2} u}{\partial y^{2}}\right) \\
& y: \quad u \frac{\partial v}{\partial x}+v \frac{\partial v}{\partial y}=-\frac{\partial p}{\partial y}+\frac{1}{\operatorname{Re}}\left(\frac{\partial^{2} v}{\partial x^{2}}+\frac{\partial^{2} v}{\partial y^{2}}\right)
\end{aligned}
$$


Energy balance:

$$
u \frac{\partial T}{\partial x}+v \frac{\partial T}{\partial y}=\frac{1}{\operatorname{Re}}\left(\frac{\partial^{2} T}{\partial x^{2}}+\frac{\partial^{2} T}{\partial y^{2}}\right)
$$

Stream function:

$$
\mathrm{u}=\frac{\partial \varphi}{\partial y}, v=-\frac{\partial \varphi}{\partial x}
$$

Vorticity equation:

$$
w=\left(\frac{\partial v}{\partial x}-\frac{\partial u}{\partial y}\right)=-\left(\frac{\partial^{2} \varphi}{\partial x^{2}}+\frac{\partial^{2} \varphi}{\partial y^{2}}\right)
$$

\section{Vorticity Transport}

Partially differentiate (2) w.r.t. y, and (3) w.r.t. x, and then by subtraction, we get the equation (7) with substituting equation (6)

$$
u \frac{\partial \omega}{\partial x}+v \frac{\partial \omega}{\partial y}=\frac{1}{\operatorname{Re}}\left(\frac{\partial^{2} \omega}{\partial x^{2}}+\frac{\partial^{2} \omega}{\partial y^{2}}\right)
$$

From the above basic equations, governing equations (1) (7) could be a classified as follow:

$$
\begin{gathered}
u \frac{\partial \omega}{\partial x}+v \frac{\partial \omega}{\partial y}=\frac{1}{\operatorname{Re}}\left(\frac{\partial^{2} \omega}{\partial x^{2}}+\frac{\partial^{2} \omega}{\partial y^{2}}\right) \\
u \frac{\partial T}{\partial x}+v \frac{\partial T}{\partial y}=\frac{1}{P e}\left(\frac{\partial^{2} T}{\partial x^{2}}+\frac{\partial^{2} T}{\partial y^{2}}\right) \\
u=\frac{\partial \varphi}{\partial x} \quad v=-\frac{\partial \varphi}{\partial x} \\
-\omega=\left(\frac{\partial^{2} \varphi}{\partial x^{2}}+\frac{\partial^{2} \varphi}{\partial y^{2}}\right)
\end{gathered}
$$

Boundary conditions:

$$
\begin{aligned}
x=0, x & =1: \\
\omega & =-\frac{1}{\operatorname{Re}} \frac{\partial^{2} \omega}{\partial x^{2}} \quad(T-T \infty)=-N u \frac{\partial T}{\partial x} \\
y=0, y & =1: \\
\omega & =-\frac{1}{\operatorname{Re}} \frac{\partial^{2} \omega}{\partial y^{2}} \quad(T-T \infty)=-N u \frac{\partial T}{\partial y}
\end{aligned}
$$

$\varphi=-\int \mathrm{V}_{\mathrm{n}} \mathrm{d} \Omega, \quad \mathrm{V}_{\mathrm{n}}$ : the normal velocity along domain boundary, $\mathrm{d} \Omega$ : domain boundary.

\section{DERIVATION OF FINITE DIFFERENCE}

The first order derivatives with upwind scheme are expressed as:

$$
A_{i j} \frac{\partial \varphi_{i j}}{\partial x_{i}}=\frac{\left(A_{i j}-\left|A_{i j}\right|\right)\left(\varphi_{i+1, j}-\varphi_{i j}\right)}{2 \Delta x_{i}}+\frac{\left(A_{i j}+\left|A_{i j}\right|\right)\left(\varphi_{i j}-\varphi_{i-1, j}\right)}{2 \Delta x_{i}}+O\left(\Delta X_{i}^{2}\right)
$$

The second order derivatives with central- difference scheme are as follows:

$$
A_{i j} \frac{\partial^{2} \phi_{i j}}{\partial x_{i}^{2}}=A_{i j} \frac{\phi_{i+1 j}-2 \phi_{i j}+\phi_{i-1 j}}{\Delta x_{i}^{2}}+O\left(\Delta X_{i}^{2}\right)
$$

To accelerate the convergent rate, a time derivative term is added into the equations (8) and (9)

$$
\frac{\partial \phi}{\partial t}=\frac{\phi_{i j}^{n+1}-\phi_{i j}^{n}}{\Delta t}
$$

After substituting the above expressions into (8), (9) respectively, algebra equations will be derived and rewritten as:

$$
A \phi_{i j}=a \phi_{i+1, j}+b \phi_{i-1, j}+c \phi_{i, j+1}+d \phi_{i j-1}+S
$$

Invoking implicit method and relax coefficient $\alpha$, we can further write:

$$
\begin{aligned}
A \phi_{i j}{ }^{n+1}= & \left(a \phi^{n}{ }_{i+1, j}+b \phi^{n+1}{ }_{i-1, j}+c \phi^{n}{ }_{i, j+1}+d \phi_{, i j-1}^{n+1}+S\right) \\
\alpha & +(1-\alpha) \phi_{i j}{ }^{n} A \\
a & =\left(\frac{1}{\operatorname{Re} \Delta x^{2}}-\frac{\left(u_{i j}-\left|u_{i j}\right|\right)}{2 \Delta x}\right) \\
b & =\left(\frac{1}{\operatorname{Re} \Delta x^{2}}+\frac{\left(u_{i j}+\left|u_{i j}\right|\right)}{2 \Delta x}\right) \\
c & =\left(\frac{1}{\operatorname{Re} \Delta y^{2}}-\frac{\left(v_{i j}-\left|v_{i j}\right|\right)}{2 \Delta y}\right) \\
d & =\left(\frac{1}{\operatorname{Re} \Delta y^{2}}+\frac{\left(v_{i j}+\left|v_{i j}\right|\right)}{2 \Delta y}\right) \\
A & =\left(\frac{2}{\operatorname{Re} \Delta x^{2}}+\frac{2}{\operatorname{Re} \Delta y^{2}}+\frac{\left|u_{i j}\right|}{\Delta x}+\frac{\left|v_{i j}\right|}{\Delta y}+1\right) \\
S & =\frac{\phi_{i}^{t-1}}{\Delta t} \\
\phi & =\omega \text { or } t
\end{aligned}
$$

where $\mathrm{n}+1$ denotes present iteration, $\mathrm{n}$ is last iteration at this time step, $\alpha$ is relax coefficient $0.5 \sim 1.2$ and t -1 is last time step. The coefficients in discretized stream equation (11), referred to Poisson equation, can be also expressed as

$$
\begin{aligned}
& a=\frac{1}{\Delta x^{2}}=b \\
& c=\frac{1}{\Delta y^{2}}=d \\
& A=2\left(\frac{1}{\Delta x^{2}}+\frac{1}{\Delta y^{2}}\right) \\
& S=\omega_{I J} \\
& \varphi=\psi
\end{aligned}
$$




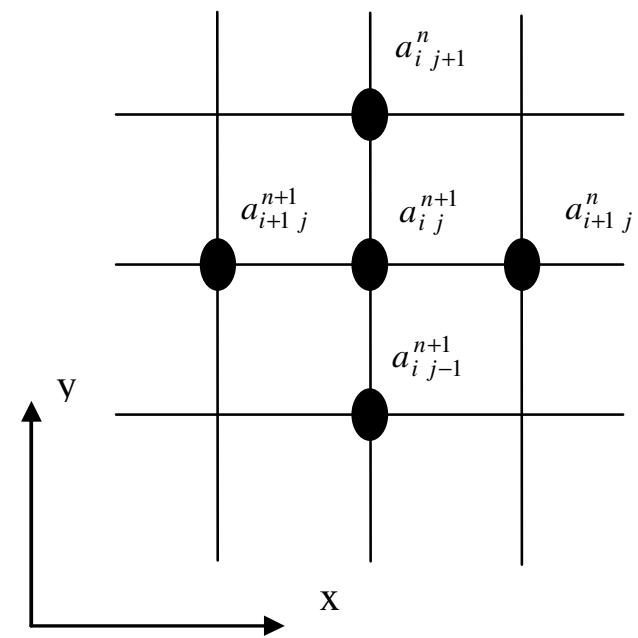

Fig. 1. The calculating distribution of grids.

The key steps for solving problem:

(1) Given the initial values of $\mathrm{u}, v, \omega, \phi, \mathrm{T}$.

(2) Specify the bounding values of $\omega, \phi, T$.

(3) Calculate vorticity $\omega$ over domain.

(4) If the relative error $\left|\frac{\omega_{i j}^{n+1}-\omega_{i j}^{n}}{\omega_{i j}^{n}}\right|>0.003$, update $\omega_{i j}^{n}=\omega_{i j}^{n+1}$ and repeat steps (3), (4).

(5) Estimate stream function $\phi$ over domain and compare the relative error $\left|\frac{\varphi_{i j}^{n+1}-\varphi_{i j}^{n}}{\varphi_{i j}^{n}}\right|$, if the ratio $>0.003$, update $\varphi_{i j}^{n}=\varphi_{i j}^{n+1}$ and repeat step (5).

(6) Determine the latest iterative values of $u, v$ over all domain.

(7) Compare the latest $u, v$ values and last $u, v$ values in domain If the relative error $\left|\frac{u_{i j}^{n+1}-u_{i j}^{n}}{u_{i j}^{n}}\right|>0.003$ or $\left|\frac{v_{i j}^{n+1}-v_{i j}^{n}}{v_{i j}^{n}}\right|>0.003$, update $u_{i j}^{n}=u_{i j}^{n+1}, v_{i j}^{n}=v_{i j}^{n+1}$ in the domain and repeat steps (3) (7).

Solve the energy equation $\mathrm{T}$ and compare the relative error. If the error $>0.003$, update $T_{i j}^{n}=T_{i j}^{n+1}$ and repeat the iterative process (8).

\section{RESULTS AND DISCUSSION}

1. By the vorticity transform method, moment equation (8) and energy equation (9) can be transferred into the expression (14) except the variables $\omega$ and $\mathrm{T}$ in respective equation. That provides a special advantage in finite difference procedure because only one discretized equation (14) with the same coefficient a,b,c,d (16) is sufficient to handle the above equations. Meanwhile, the discretized equation can be also fitted in the stream-vorticity equation by changing the value in coefficient $a, b, c, d$ and source term $S$ directly.

2. In both the implicit and explicit iterations, the value at given grid is calculated by averaging those at its four neighboring corners. The difference is that in explicit iteration, the neighboring values at given point was estimated from last iteration; but in the implicit iteration, the values at the top corner and right corner were obtained from the last iteration and those at the left and bottom corner are gotten from present iteration values as shown in Fig. 1. Therefore it is faster to use the implicit iteration to get the value at a given grid.

3. Sufficient condition for convergence proposed by Patankar(1970):

Rule 1: consistency at control-volume force

Rule 2: positive efficient $>a, b, c, d>0$

Rule 3: $\left|\mathrm{a}_{i i}\right| \geq \sum_{\substack{j=1 \\ j \neq i}}^{n}\left|\mathrm{a}_{i i}\right|$

$>\mathrm{A}>\mathrm{a}+\mathrm{b}+\mathrm{c}+\mathrm{d}$

Rule 4: negative-slope linearization of source term $>\mathrm{S}=\mathrm{Sc}+\mathrm{SpTp}$, the coefficient $\mathrm{Sp}$ must always be less than or equal to zero.

For present model, we observe that the coefficients A, a, b, $\mathrm{c}$ and $\mathrm{d}$ in the central difference equations, whether in case of vorticity transport or energy equation, are all positive and $\mathrm{A}>\mathrm{a}+\mathrm{b}+\mathrm{c}+\mathrm{d}$. This satisfies the convergent condition of the Gauss-Seidel's algorithm and Patankar's rule. Therefore in the course of computation,we use both the central difference and upward scheme that make the iterated values of $\mathrm{u}, v$ and $\mathrm{T}$ approach to a stable without over restricted the grid size $d x$, dy and Re. Hence Re value can be extended in the allowable range of computation until the model begins to show rather large error when $\mathrm{Re}$ value reaches 5000 .

4. Though the first order truncation error $(\Delta \mathrm{X}, \Delta \mathrm{Y})$ is derived in the upwind scheme method, the smaller step size $\Delta \mathrm{X}, \Delta \mathrm{Y}$ with increased grids which are necessary to guarantee the more accurate solution will be further examined in the grids test. Grids $20 \times 30,30 \times 45,40 \times 60$ were selected to uniformly distribute in the calculating domain. After comparison with individual result to all corresponding grids in domain, their maximum relative error will be not more than $3 \%$ and the simulating result shows that the adopt of grids $20 \times 30$ has been adequate to describe the convective motion without losing the overall behavior.

5. It usually specifies a uniform distribution of inlet or outlet flow velocity on the boundary walls as shown in Fig. 2 which just likes a Dirac function applied on wall. Therefore the discontinuous value and derivative observed at $\mathrm{X}_{1}$ and $\mathrm{X}_{2}$ will result in a divergent solution in the end or unreasonable solution distributed around the inlet and outlet; even the result simulated is just fitted in the low Reynold number case. To improve above defect, the adopt of streamline function with a integration of flow velocity on 


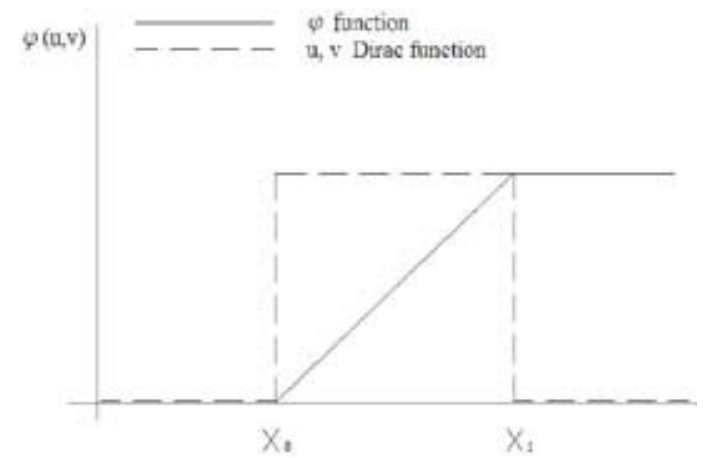

Fig. 2. The illustration of $\psi$ and $u, v$ Dirac boundary condition.

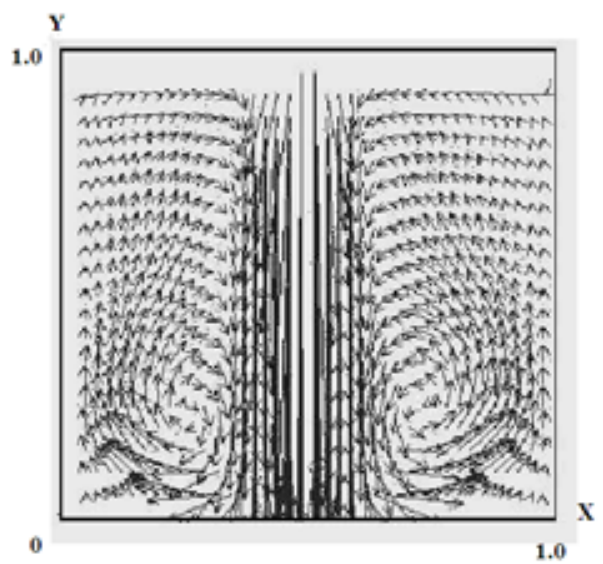

Fig. 3. Simulation of flow velocity distribution with $\mathrm{Re}=2500$ in resent simulation code.

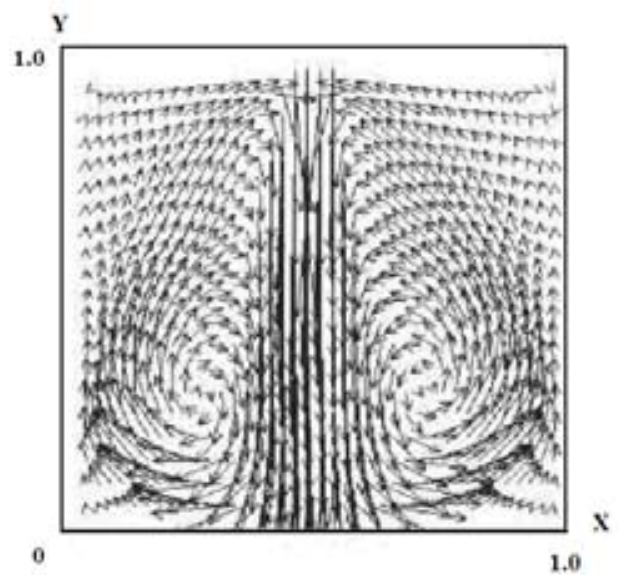

Fig. 4. Simulation of flow velocity distribution with $R e=2500$ by CFD package "NISTIR."

boundary surface will be demonstrated in Fig. 2 where the trouble caused by the discontinuous phenomena can be avoided by a continuous streamline function with integration skills and a more realistic solution that satisfied the first convergent rule proposed by Partankar will be predicted.

6. Under the dimensionless parameters $\mathrm{Re}=2500, \mathrm{Pe}=25$ are considered, both present codes with $\phi$ continuous boundary conditions in present code (see Fig. 3) and CFD package "NISTIR" with the Dirac function of $u, v$ specified at the boundary (see Fig. 4) display a similar flow distribution

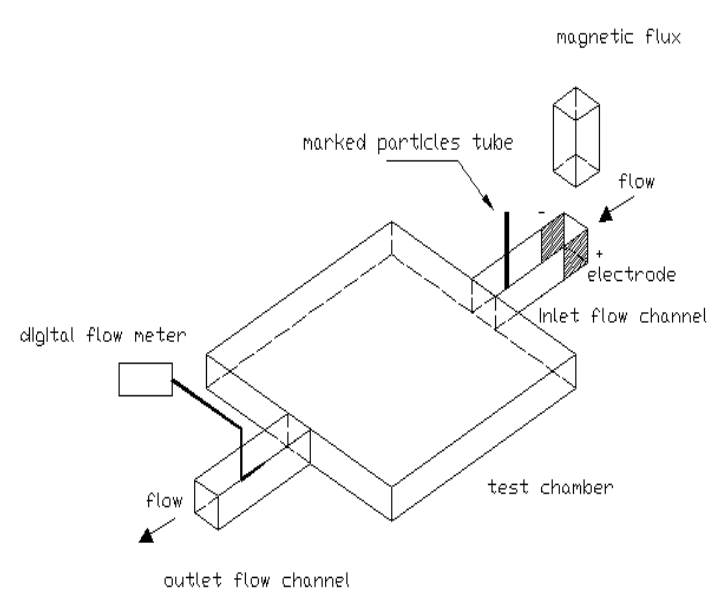

Fig. 5. Schematic view of experimental apparatus.

Table 1. Parameters for experimental apparatus.

\begin{tabular}{ll} 
Parameter & Value \\
\hline Reservoir dimension $\left(\mathrm{M}^{3}\right)$ & $0.1 \times 0.1 \times 0.01$ \\
L type copper sheet $\left(\mathrm{M}^{2}\right)$ & $0.01 \times 0.01$ \\
Inlet, outlet flow channel $\left(\mathrm{M}^{3}\right)$ & $0.1 \times 0.1 \times 0.5$ \\
Magnetic flux density, $B(\mathrm{~T})$ & $0.02 \sim 0.04$ \\
Input voltage, $(\mathrm{V})$ & $6 \sim 15$ \\
Working medium: $(\mathrm{NaCl}$ solution $)$ & 1058 \\
Density, $\rho\left(\mathrm{kg} / \mathrm{m}^{3}\right)$ & 1.5 \\
Conductivity, $\sigma(\mathrm{S} / \mathrm{m})$ & 0.001 \\
Viscosity, $\mu(\mathrm{Pas})$ &
\end{tabular}

in most space except the data around the inlet where the flow velocity gradient behaves a discontinuous change and seems to be unreasonable in Fig. 4. To improve above defects, the adoption of stream function in present code is available in avoiding the discontinuous phenomena near the boundary walls.

7. To verify the simulation results, an experimental procedure consisting of liquid reservoir, inlet (outlet) flow channel, MHD unit and marked particles tube were set up as shown in Fig. 5. Additional pitot tube used to measure the outlet flow velocity and connected to the digital flow meter was also equipped. All dimensions of experimental apparatus would be listed in Table 1. A MHD (magnethohydrodynamics) mechanism including magnetism and copper sheets was also set up at the inlet flow channel and taken as the driving source. When the electric power was supplied to the separate copper electrode, the induced current produced in the working medium $(\mathrm{NaCl}$ solution) would interact with the external magnetic flux to generate the Lorentz force which was perpendicular to the direction of magnetic field and induced current. As the Lorentz force began to work, the flow velocity could be measured by digital meter and then the flow stream was traced by marked particles. Corresponding to the inlet flow velocity $0.025 \mathrm{~m} / \mathrm{s}$ in experimental test, Re would be roughly estimated about 2500 


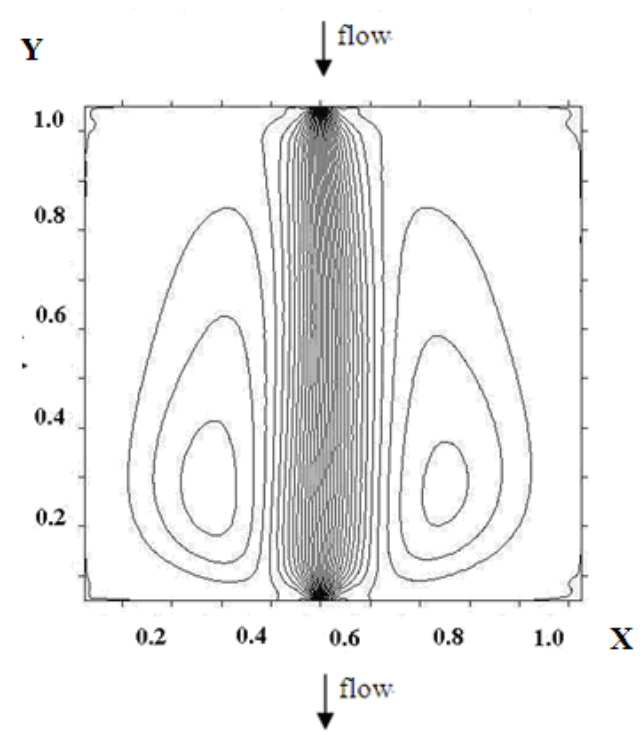

Fig. 6. Simulation of streamline distribution with $\mathrm{Re}=2500$ in present simulation code.

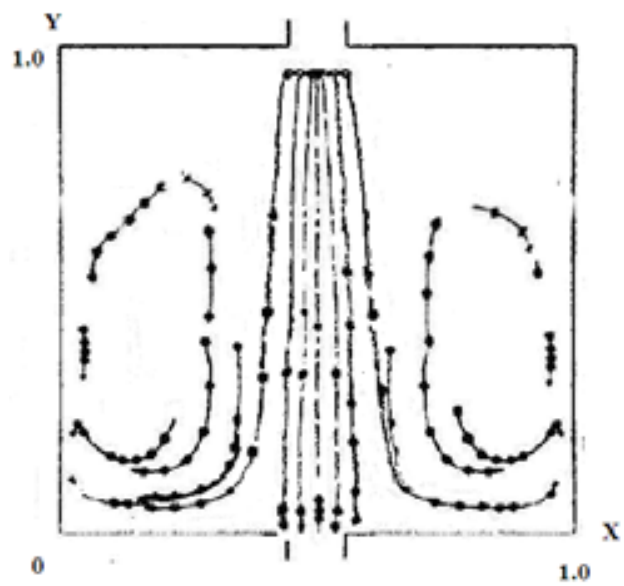

Fig. 7. Marked particles distribution of experimental photography with continuous exposure in test reservoir.

used as the input value of simulating code.

8. In dealing with the streamline distribution, both corner vortexes just symmetrical to the strip from inlet to outlet in Fig. 6 were formed as the isolated region where the fluid that could not flow out has to turn around near the outlet and circulate inside the space to obey flow conservation. Compared the image captured by continuous exposure in experimental reservoir (Fig. 7), most marked particles were concentrated at the circulated region where the vortex contour had been successfully traced. Which was close agreement with simulation results (Fig. 6) and could also identify the correct of numerical scheme used in the simulation code

9. Fig. 8 is the temperature distribution chart, where the wall temperature 0.8 and inlet, outlet temperature 0.5 were specified. Due to the temperature distribution in the vortex regions appearing at the upper and lower region in space is nearly unaffected by their neighborhood, the bad convec-

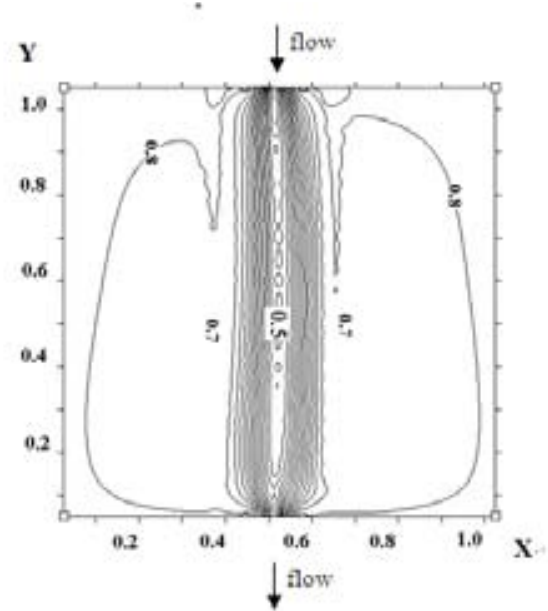

Fig. 8. Simulation of temperature distribution with $\mathrm{Re}=\mathbf{2 5 0 0}$ $\mathrm{Pe}=25$ in $\varphi$ continuous boundary value.

tion region covering isothermal region $\mathrm{T}=0.7 \sim 0.8$ will be predicted there. As to the central strip from inlet to outlet, the strong convection induced by fast flow velocity will keep constant temperature about 0.5 along the traveling path of inlet jet, which is nearly the same as temperature specified at the inlet, outlet of space. A slight away from the central strip, the isothermal contour became denser; that implies the temperature gradient will significantly vary in the normal direction of jet flow. In other words, the temperature there will quickly climb up from 0.5 to 0.7 in a very short displacement normal to the central strips.

\section{CONCLUSIONS}

1. By using the vorticity - stream transformation, the unstable iteration caused by the pressure term in 2-D Navier Stoke equations can be avoided and the mass conservation will be automatically satisfied by introducing the stream function.

2. Applying the vorticity-stream transformation, the moment and energy equations can be discretized into the same algebra equations, which gives a special advantage in numerical calculating.

3. By using the implicit iteration, it is possible to make the numerical solution more stable and the faster computation during iteration process is less restricted.

4. The mixed use of central difference and upwind scheme guarantees the difference coefficients in the vorticity transport, stream function and energy equations positive; that satisfied Gauss Seidel's convergent condition.

5. On the boundary wall, the distribution of Dirac function in $\mathrm{u}, v$ will cause the flow vector to become discontinuous around there. To overcome the unreasonable phenomena, a modification by using stream function with the integral of $\mathrm{u}, v$ on the wall makes flow distribution more realistic and approach to the experimental result.

6. Introducing boundary layer thickness to simplify the $\omega-\varphi$ equation on the boundary walls has no significant bad influence on the result. 
7. In this study, the distributions of flow velocities, stream lines and temperature simulated can be reasonably interpreted and conform to the experimental results.

\section{NOMENCLATURE}

$\mathrm{u}$ : nodimensional flow velocity in $\mathrm{x}$ direction

$v$ : nodimensional flow velocity in y direction

$\omega$ : nodimensional vorticity

$\psi$ : nodimensional stream function

$\mathrm{T}$ : nodimensional temperature

Re: Renold number

$\mathrm{Nu}$ :Nussult number

Pe: Pelect number

$\Delta \mathrm{x}$ : nodimensional $\mathrm{x}$ step side

$\Delta y$ : nodimensional y step side

\section{REFERENCES}

1. Ames, W. F., Numerical Methods for Partial Differential Equations, 2nd. ed., Academic , NewYork (1977)

2. Chilukuri, R. and Pletcher, R. H., "Numerical solutions to the partially parabolized Navier-Stokes equations for developing flow in a channel," Numerical Heat Transfer, Vol. 3, pp. 169-188 (1980).

3. Chorin, A. J., "A numerical method for solving incompressible viscous flow problems," Computational Physics, Vol. 2, pp. 12-26 (1967).

4. Hirano, H., Seo, M., and Ozoe, H., "Two-dimensional numerical computation for Rayleigh-Benard convection with both the Navier-Stokes equation and the Boltzmann equation," Modeling Simulation Material Science and Engineering, Vol. 10, pp. 765-780 (2002).

5. Kurabuchi, T., Fang, T. F., and Richard A., "A numerical method for calculating indoor air flow using a turbulence model," National Institute of Standard and Technology commercial code, Tokyo (1990).

6. Patankar, S. V. and Spalding, D. B., Heat and Mass Transfer in Boundary Layers, 2nd. ed., Intertext Books, London (1970)

7. Tzeng, P. Y. and Liu, M. H., "Direct simulation Monte Carlo modeling on two-dimensional Raleigh-Benard instabilities of rarefied gas," Numerical Heat Transfer A, Vol. 47, No. 8, pp. 805-823 (2005).

8. Warming, R. F. and Beam, R. M., "On the construction and application of implicit factored schemes for conservation laws," Proceedings of the Symposium on Computational Fluid Dynamics, New York, Vol. 11, pp. 85-129 (1977). 